East African Medical Journal Vol. 86 No. 12 December 2009

LIFE-THREATENING LITHIUM-INDUCED DIABETES INSIPIDUS AFTER COLONIC SURGERY: REPORT OF TWO CASES

W. Baraza, MRCS, Registrar in General Surgery, The University of Sheffield, Western Bank, Sheffield S10 2TN, J. P. Garner, MD, FRCSEd (GenSurg), Consultant ColorectalSurgeon, Rotherham NHSFoundation Trust, Moorgate Road, Rotherham, R. Slater, MD, FRCS (Gen Surg), Consultant Surgeon, Rotherham NHS Foundation Trust, Moorga Road, Rotherham and S. Amin, Consultant Surgeon, Northern General Hospital, Herries Road, Sheffield, The Department of Colorectal Surgery, The Northern General Hospital, Sheffield Teaching Hospitals NHS Trust, Herries Road, Sheffield S5 7AU

Request for reprints to: Mr. W. Baraza, 59 Ryegate Road, Sheffield, S10 5FB

\title{
LIFE-THREATENING LITHIUM-INDUCED DIABETES INSIPIDUS AFTER COLONIC SURGERY: REPORT OF TWO CASES
}

\author{
W. BARAZA, J. P. GARNER, R. SLATER and S. AMIN
}

\begin{abstract}
SUMMARY
Colonic surgery is associated with major disturbances to patients' fluid balance as a result of pre-operative starvation, intra-operative blood loss, insensible losses, and post-operative 'third space' losses. In the post-operative period, junior surgeons are well trained to manage oliguria, but have little experience of the management of post-operative polyuria. Nephrogenic diabetes insipidus occurs in $12 \%$ of patients on lithium therapy and may present in the immediate post-operative period with massive polyuria, profound dehydration and life threatening hypernatraemia, as patients are unable to ingest large volumes of fluid orally. We report two cases, one life threatening, of lithium induced nephrogenic diabetes insipidus (LINDI) which presented in the early postoperative period after emergency colonic surgery whilst the patients were denied oral intake. The first patient attained a peak serum sodium concentration of $185 \mathrm{mmol} / 1$ and required ventilation after a respiratory arrest. We suggest any surgical patient who describes a history of bipolar disorder or lithium therapy should be questioned directly to ascertain confirmatory features of LINDI, and have post-operative polyuria treated with aggressive fluid replacement.
\end{abstract}

\section{INTRODUCTION}

Accurate fluid balance and maintenance of an acceptable urine output by intravenous fluid therapy is a cornerstone of good perioperative surgical care and is well known to junior surgeons throughout the world. Although surgical texts and courses $(1,2)$ cover the management of post-operative oliguria in detail, there is little in the surgical literature about the management of postoperative polyuria. Nephrogenic diabetes insipidus, when the kidney cannot concentrate the urine, is a common cause of polyuriait may be inherited or acquired, with the commonest acquired aetiology being the previous or continuing use of lithium salts, commonly prescribed as a moodstabilising agent in bipolar disorder. Lithium-induced nephrogenic diabetes insipidus (LINDI) occurs in up to $12 \%$ of patients taking lithium and may persist even after withdrawal of the treatment $(3,4)$. Patients typically compensate by drinking huge quantities of fluids in what becomes a normal part of daily life for them. Failure of surgical staff to recognise the potential for profound perioperative dehydration in LINDI because of restriction of oral fluid intake may have life-threatening consequences from hypernatraemia, hyperosmolar coma or cardiovascular collapse. We present two cases of LINDI in surgical patients, one of which was life-threatening, and discuss the management of this uncommon condition and how these complications may be avoided.

\section{CASE REPORTS}

Case 1: A 59 year old woman was admitted as an emergency with large bowel obstruction from a sigmoid adenocarcinoma. She was previously fit and well apart from hypothyroidism and bipolar disorder for which she had taken lithium carbonate for more than 20 years. At laparotomy, a left hemicolectomy with end to end anastomosis and a left salpingo-oophorectomy for an incidental ovarian mass was performed. Within 24 hours of surgery she was confused and had passed $7000 \mathrm{ml}$ of dilute urine, her serum sodium had risen to $176 \mathrm{mmol} / 1$ with a normal serum potassium level and she had an elevated serum creatinine of $215 \mathrm{mmol} / 1$. She was receiving no fluid orally and had received only $2700 \mathrm{mls}$ of intravenous 
crystalloid (2 litres of Ringer's lactate and $700 \mathrm{ml}$ $0.9 \%$ sodium chloride solution) as replacement. Her lithium levels were within the therapeutic range. Unsure of the cause of the patient's confusion and worsening fluid and electrolyte status an urgent medical consultation was organised and a diagnosis of LINDI was made; rehydration with $0.9 \%$ saline at $250 \mathrm{ml} / \mathrm{hr}$ was commenced after transfer to the High Dependency Unit (HDU). During the following day she remained confused and her sodium rose to $185 \mathrm{mmol} / \mathrm{l}$, serum osmolality was $391 \mathrm{mOsm} / \mathrm{kg}$ (normal range 282-295) and urine osmolality of 166 $\mathrm{mOsm} / \mathrm{kg}$ (normal range 500-800). She continued to produce $100-350 \mathrm{ml} / \mathrm{hr}$ of urine and her calculated free water deficit was 8.5 litres over 48 hours. A trial of subcutaneous desmopressin ( $2 \mathrm{mcg}$ once daily) was unsuccessful in reducing the volume of polyuria, and fluid replacement continued with $0.18 \%$ saline $/ 5 \%$ dextrose solution.

On the fifth post-operative day she suffered a respiratory arrest and required intubation and ventilation for 48 hours. Over this period her serum electrolytes returned to normal with continued $0.18 \%$ saline $/ 5 \%$ dextrose solution resuscitation. After extubation she resumed oral intake and on direct questioning admitted to routinely drinking 2-3 litres of carbonated drinks each night to try and quench her thirst. She was discharged on day 17 with an ongoing polydipsia and high volume oral fluid intake. She remained well when reviewed in clinic six weeks later with normal serum urea and electrolytes and a mildly raised serum osmolality of $317 \mathrm{mOsm} / \mathrm{kg}$. Her serum sodium concentration and urine outputover the course of her admission are shown in Figure 1.

Figure 1

Serum sodium trend, Case 1

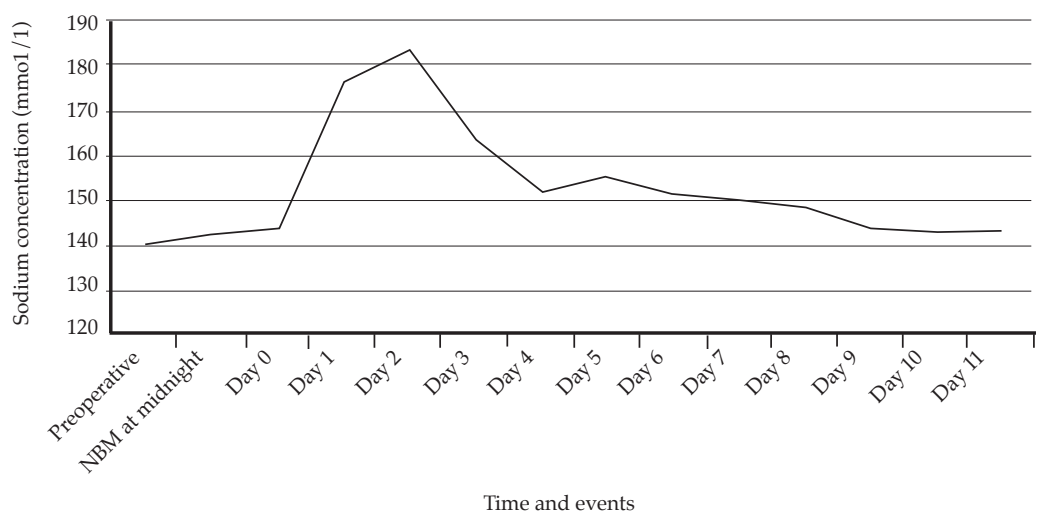

Figure 2

Mean urine output, Case 1

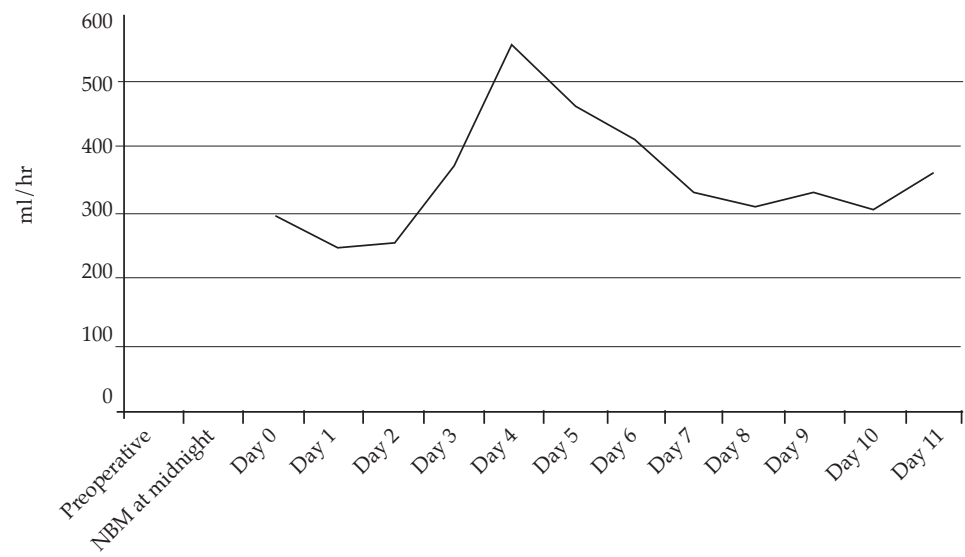

Time and events

Legends

Events

Day 1: Rehydration with $0.9 \%$ saline 6 hourly

Day 2: Rehydration with 5\% dextrose, trial of 2 micrograms DDAVP

Day 3: Rehydration with 5\% dextrose specifically replacing urinary losses

Day 4: Trial of 2 micrograms DDAVP; respiratory arrest due to mucus plug

Day 6: Enteral hydration via nasogastric tube from $25 \mathrm{ml} / \mathrm{hr}$ and gradual build-up to free oral intake 
Case 2: A 71 year old lady was admitted as an emergency with a history of constipation, severe abdominal pain and distension resulting in an emergency sigmoid colectomy for perforated sigmoid volvulus. She had a past medical history of mild chronic renal failure and bipolar disorder previously treated with lithium which had been discontinued many years prior to her presentation. She also took low dose desmopressin for previously suspected LINDI. Despite a planned admission to the High Dependency Unit (HDU) post-operatively, she still developed polyuria within 48 hours of ceasing oral intake (urine output $200-350 \mathrm{ml} /$ hour) and a 2 litre fluid deficit. Her serum sodium was $151 \mathrm{mmol} / 1$ with a mild hyperchloraemic acidosis, serum osmolality of $322 \mathrm{mOsm} / \mathrm{kg}$ and urine osmolality of $173 \mathrm{mOsm} / \mathrm{kg}$. Ringer's lactate infusion was changed to $5 \%$ dextrose and her desmopressin increased to $1 \mathrm{mcg}$ four times daily subcutaneously and then $10 \mathrm{mcg}$ four times daily intranasally without appreciable effect. Two days later her serum sodium remained at $151 \mathrm{mmol} / 1$ and she remained polyuric ( $>6000 \mathrm{ml} /$ day), but she had recommenced oral fluid intake. By the eighth postoperative day her serum sodium had normalised; she was discharged well but polyuric five weeks after admission.

\section{DISCUSSION}

Polyuria and secondary polydipsia producing huge volumes of unconcentrated urine with a resultant serum concentration and hypernatraemia are the hallmarks of nephrogenic diabetes insipidus. It may rarely be due to inherited conditions but is usually acquired, most commonly from lithium therapy.

Lithium salts have long been recognised as a cause of nephrogenic diabetes insipidus by rendering the distal tubule and collecting duct of the nephron completely, or more usually partially, insensitive to the concentrating action of vasopressin. They also downregulate aquaporins, integral membrane proteins that are 'pores' through which water is normally resorbed $(5,6)$. These channels are impermeable to ions and are, in the healthy state, regulated by endogenous vasopressin. The effects of lithium on the tubule may persist for many years following its cessation and may be due to the induction of chronic glomerular and interstitial nephropathies (7).

The diagnosis of LINDI depends on a careful history and examination as well as the logical measurement of biochemical parameters in response to water deprivation and vasopressin administration. The relevant drug history confirms lithium therapy as well as possible polydipsia and polyuria. A positive water deprivation test is then performed which confirms the patient's inability to concentrate urine suggesting diabetes insipidus. This involves measuring patients' weight, electrolytes, and serum and urine osmolalities before and after depriving them of water for a variable period of time (usually 12 hours). In the healthy state, urine osmolality should at least double. Following this, the aetiology of the diabetes insipidus is determined by demonstrating the patient's sensitivity to vasopressin. In addition to a negative water deprivation test, patients with LINDI typically have a less than $10 \%$ increase in urine osmolality after a trial of vasopressin (8).

The treatment of established LINDI is restitution of intravascular volume whilstattempting to stimulate renal concentration of urine. Hypernatraemia, which is initially due to serum concentration from the loss of free water, is potentially exacerbated by resuscitation with normal saline, as the kidney cannot eliminate the additional sodium load (6). Dextrose or dextrose saline solution is the intravenous fluid of choice whilst hypernatraemia persists.

As the underlying pathophysiology is a partial or complete insensitivity to endogenous vasopressin, the administration of exogenous vasopressin analogues may only be beneficial in those cases where insensitivity is partial - it had no effect in either of our cases. Other pharmacological interventions include the use of non-steroidal antiinflammatory drugs (NSAIDS) and, paradoxically, diuretics. NSAIDs reduce prostaglandin-mediated glomerular filtration and hence reduce free water loss $(6,9)$. The exact mechanisms by which thiazide diuretics and amiloride act in LINDI is unclear. Amiloride may decrease the effect of lithium on the collecting tubules and render them more sensitive to vasopressin $(10,11)$. Thiazide diuretics may decrease polyuria by increasing renal sodium losses, contracting extracellular fluid volume and decreasing the glomerular filtration rate. Furthermore, both thiazide diuretics and amiloride may increase the expression of aquaporins and sodium channels in the distal tubule, enhancing water resorption $(5,12,13)$.

Most patients with LINDI, aware of the diagnosis or not, maintain health by regularly drinking abnormally large volumes of fluid, which becomes such a part of their daily life that they may well not volunteer this information during routine surgical clerking. We suggest that any patient who gives a history of past or present lithium therapy, or bipolar disorder that may have previously been treated with lithium, should be asked directly about symptoms of polyuria and polydipsia. Pre-operative recognition of the condition should prompt more aggressive intravenous fluid replacement for these patients when denied oral fluids, more frequent estimation of serum electrolytes and closer peri-operative monitoring to reduce the risks of hypernatraemia and hypersomolar coma.

In conclusion, a history of bipolar disorder or lithium therapy should necessitate direct enquiry 
about the presence of polyuria and polydipsia to avoid postoperative complications. Unexpected post-operative polyuria should prompt the early consideration of a diagnosis of LINDI and institution of aggressive intravenous rehydration, using dextrose solutions in the face of hypernatraemia. In the established cases of LINDI, desmopressin may be ineffective and the use of amiloride, thiazide diuretics or NSAIDs should be considered.

\section{REFERENCES}

1. Care of the Critically ill Surgical Patient Course Manual. The Royal College of Surgeons of England.

2. Clinical Surgery in General, RCS Course Manual (4th Edition edn). Churchill Livingstone, 2004.

3. Hyperosmolar coma due to lithium-induced diabetes insipidus. Lancet. 1995; 346: 413-417.

4. Thompson, C. J., France, A. J. and Baylis, P. H. Persistent nephrogenic diabetes insipidus following lithium therapy. Scott. Med. J. 1997; 42: 16-17.

5. Kim, G. H., Lee, J. W., Oh Y. K. et al. Antidiuretic effect of hydrochlorothiazide in lithium-induced nephrogenic diabetes insipidus is associated with upregulation of aquaporin-2, $\mathrm{Na}-\mathrm{Cl}$ co-transporter, and epithelial sodium channel. J. Am. Soc. Nephrol. 2004; 15: 2836-2843.
6. Allen, H. M., Jackson, R. L., Winchester, M. D., Deck, L.V. and Allon M. Indomethacin in the treatment of lithium-induced nephrogenic diabetes insipidus. Arch. Intern. Med. 1989; 149: 1123-1126.

7. Markowitz, G. S., Radhakrishnan, J., Kambham, N. et al. Lithium nephrotoxicity: a progressive combined glomerular and tubulointerstitial nephropathy.J. Am. Soc. Nephrol. 2000;11: 1439-1448.

8. Stone, K. A. Lithium-induced nephrogenic diabetes insipidus. J. Am. Board. Fam. Pract. 1999;12: 43-47.

9. Lam, S.S. and Kjellstrand, C. Emergency treatment of lithium-induced diabetes insipidus with nonsteroidal anti-inflammatory drugs. Ren. Fail. 1997;19: 183188.

10. Batlle, D. C., von Riotte, A. B., Gaviria, M. and Grupp, M. Amelioration of polyuria by amiloride in patients receiving long-term lithium therapy. N. Engl. J. Med. 1985; 312: 408-414.

11. Bedford, J. J., Weggery, S., Ellis G, et al. Lithiuminduced nephrogenic diabetes insipidus: Renal effects of amiloride. Clin. J. Am. Soc. Nephrol. 2008; 3: 1324-1331.

12. Loffing, J. Paradoxical antidiuretic effect of thiazides in diabetes insipidus: another piece in the puzzle. J. Am. Soc. Nephrol. 2004;15: 2948-2950.

13. Nguyen, M. K., Nielsen, S. and Kurtz, I. Molecular pathogenesis of nephrogenic diabetes insipidus. Clin. Exp. Nephrol. 2003; 7: 9-17. 